\title{
Asymptotics for minimal overlapping patterns for generalized Euler permutations, standard tableaux of rectangular shapes, and column strict arrays
}

\author{
Ran $\operatorname{Pan}^{1}$ \\ Jeffrey B. Remmel ${ }^{2}$ \\ 1 Department of Mathematics, UCSD La Jolla, CA, 92093-0112, rlpan@ucsd.edu \\ 2 Department of Mathematics, UCSD La Jolla, CA, 92093-0112, jremmel@ucsd.edu
}

received $29^{\text {th }}$ Oct. 2015, revised $26^{\text {th }}$ Feb. 2016, accepted 24th Mar. 2016.

\begin{abstract}
A permutation $\tau$ in the symmetric group $S_{j}$ is minimally overlapping if any two consecutive occurrences of $\tau$ in a permutation $\sigma$ can share at most one element. Bóna showed that the proportion of minimal overlapping patterns in $S_{j}$ is at least $3-e$. Given a permutation $\sigma$, we let $\operatorname{Des}(\sigma)$ denote the set of descents of $\sigma$. We study the class of permutations $\sigma \in S_{k n}$ whose descent set is contained in the set $\{k, 2 k, \ldots(n-1) k\}$. For example, up-down permutations in $S_{2 n}$ are the set of permutations whose descent equal $\sigma$ such that $\operatorname{Des}(\sigma)=\{2,4, \ldots, 2 n-2\}$. There are natural analogues of the minimal overlapping permutations for such classes of permutations and we study the proportion of minimal overlapping patterns for each such class. We show that the proportion of minimal overlapping permutations in such classes approaches 1 as $k$ goes to infinity. We also study the proportion of minimal overlapping patterns in standard Young tableaux of shape $\left(n^{k}\right)$.
\end{abstract}

Keywords: permutations, arrays, minimal overlapping patterns

\section{Introduction}

Let $S_{n}$ denote the set of permutations of $[n]=\{1, \ldots, n\}$. We let $k[n]=\{k, 2 k, \ldots, n k\}$ if $k, n \geq 1$. If $\sigma=\sigma_{1} \ldots \sigma_{n}$ is an element of $S_{n}$, we let $\operatorname{Des}(\sigma)=\left\{i: \sigma_{i}>\sigma_{i+1}\right\}$. For any $k, n \geq 1, \mathcal{C}_{=k[n-1]}$ denote the set of all $\sigma$ in $S_{k n}$ such that $\operatorname{Des}(\sigma)=k[n-1]$ and $\mathcal{C}_{\subseteq k[n-1]}$ denote the set of all $\sigma$ in $S_{k n}$ such that $\operatorname{Des}(\sigma) \subseteq k[n-1]$. For example, elements of $\mathcal{C}_{=2[n-1]}$ are permutations $\sigma=\sigma_{1} \sigma_{2} \ldots \sigma_{2 n} \in S_{2 n}$ such that

$$
\sigma_{1}<\sigma_{2}>\sigma_{3}<\sigma_{4}>\cdots .
$$

These are called up-down permutations or even alternating permutations.

One way to think of elements in $\mathcal{C}_{\subseteq k[n-1]}$ is as column strict arrays which were studied by Harmse and Remmel in [6] and [7]. A column-strict array $P$ is a filling of a $k \times n$ rectangular array with $1,2, \cdots, k n$ such that elements increase from bottom to top in each column. Let $\mathcal{F}_{n, k}$ denote the set of all the columnstrict arrays with $n$ columns and $k$ rows. Given a $P \in \mathcal{F}_{n, k}$, we let $P[i, j]$ denote element in the $i$ th

ISSN $1365-8050 \quad$ (c) 2016 by the author(s)

Distributed under a Creative Commons Attribution 4.0 International License 
column and $j$ th row where the columns are labeled from left to right and the rows are labeled from bottom to top. We let $\sigma_{P}$ denote the permutation

$$
\sigma_{p}=P[1,1] P[1,2] \ldots P[1, k] P[2,1] P[2,2] \ldots P[2, k] \ldots P[n, 1] P[n, 2] \ldots P[n, k] .
$$

For example, if $P$ is the element of $\mathcal{F}_{4,3}$ pictured in Figure 1 , then $\sigma_{P}=311121246810579$. This given, it is easy to see that $\sigma_{P} \in \mathcal{C}_{=k[n-1]}$ if and only if $P[i, k]>P[i+1,1]$ for all $1 \leq i \leq n-1$. An array $P \in \mathcal{F}_{n, k}$ is a standard tableau of shape $\left(n^{k}\right)$ if $P$ is strictly increasing in rows, reading from left to right. That is, $P$ is strictly increasing in rows if for all $1 \leq j \leq k$,

$$
P[1, j]<P[2, j]<\cdots<P[n, j] .
$$

We let $\mathcal{S} \mathcal{T}\left(n^{k}\right)$ denote the set of all standard tableaux of shape $\left(n^{k}\right)$.

\begin{tabular}{|c|c|c|c|}
\hline 12 & 4 & 10 & 9 \\
\hline 11 & 2 & 8 & 7 \\
\hline 3 & 1 & 6 & 5 \\
\hline
\end{tabular}

Fig. 1: An element of $\mathcal{F}_{4,3}$.

Given any sequence of pairwise distinct positive integers, $\alpha=\alpha_{1} \ldots \alpha_{n}$, we let $\operatorname{red}(\alpha)$ denote the permutation of $S_{n}$ that results from $\alpha$ by replacing the $i$ th smallest element of $\alpha$ by $i$ for $i=1, \ldots, n$. For example, $\operatorname{red}\left(\begin{array}{llll}3 & 6 & 8 & 2\end{array}\right)=23415$. Given a permutation $\tau \in S_{j}$ and permutation $\sigma=\sigma_{1} \ldots \sigma_{n} \in S_{n}$, we say that

1. $\tau$ occurs in $\sigma$ if there are $1 \leq i_{1}<i_{2}<\cdots<i_{j} \leq n$ such that $\operatorname{red}\left(\sigma_{i_{1}} \ldots \sigma_{i_{j}}\right)=\tau$,

2. $\sigma$ avoids $\tau$ if there is no occurrence of $\tau$ in $\sigma$, and

3. there is a $\tau$-match in $\sigma$ starting at position $i$ if $\operatorname{red}\left(\sigma_{i} \sigma_{i+1} \ldots \sigma_{i+j-1}\right)=\tau$.

We let $\tau$ - $\operatorname{mch}(\sigma)$ denote the number of $\tau$-matches in $\sigma$. We say that $\tau$ has the minimal overlapping property or $\tau$ is minimally overlapping if the smallest $n$ such that there exists $\sigma \in S_{n}$ such that $\tau-\operatorname{mch}(\sigma)=2$ is $2 j-1$. This means in any two consecutive $\tau$-matches in a permutation $\sigma$ can share at most one element which must necessarily be at the end of the first $\tau$-match and the start of the second $\tau$-match. It follows that if $\tau \in S_{j}$ is minimally overlapping, then the smallest $n$ such that there exist a $\sigma \in S_{n}$ such that $\tau$-mch $(\sigma)=k$ is $k(j-1)+1$. We call these particular elements of $S_{k(j-1)+1}$ maximum packings for $\tau$ and we let $\mathcal{M P}_{\tau, k(j-1)+1}=\left\{\sigma \in S_{k(j-1)+1}: \tau\right.$-mch $\left.(\sigma)=k\right\}$. We let $\mathrm{mp}_{\tau, k(j-1)+1}=\left|\mathcal{M P}_{\tau, k(j-1)+1}\right|$

Minimal overlapping permutations are nice in that we have a nice expression for the generating function of $x^{\tau-\operatorname{mch}(\sigma)}$ over permutations. That is, Duane and Remmel [3] proved the following theorem.

Theorem 1. If $\tau \in S_{j}$ has the minimal overlapping property, then

$$
\begin{aligned}
& \sum_{n \geq 0} \frac{t^{n}}{n !} \sum_{\sigma \in S_{n}} x^{\tau-m c h(\sigma)} p^{\operatorname{coinv}(\sigma)} q^{\operatorname{inv}(\sigma)}= \\
& \frac{1}{1-\left(t+\sum_{n \geq 1} \frac{t^{n(j-1)+1}}{[n(j-1)+1]_{p, q} !}(x-1)^{n} m p_{\tau, n(j-1)+1}(p, q)\right)} .
\end{aligned}
$$


Here if $\sigma=\sigma_{1} \ldots \sigma_{n} \in S_{n}$, then $\operatorname{inv}(\sigma)=\left|\left\{(i, j): 1 \leq i<j \leq n \& \sigma_{i}>\sigma_{j}\right\}\right|$ and $\operatorname{coinv}(\sigma)=$ $\left|\left\{(i, j): 1 \leq i<j \leq n \& \sigma_{i}<\sigma_{j}\right\}\right|$ and

$$
\operatorname{mp}_{\tau, n(j-1)+1}(p, q)=\sum_{\sigma \in \mathcal{M P}_{\tau, n(j-1)+1}} p^{\operatorname{coinv}(\sigma)} q^{\operatorname{inv}(\sigma)}
$$

Harmse and Remmel generalized these definitions and results to $\mathcal{F}_{n, k}$. However, as we shall see their definitions make perfectly good sense in the the setting of $\mathcal{C}_{=k[n-1]}$ or $\mathcal{S T}\left(n^{k}\right)$. That is, if $F$ is any filling of a $k \times n$-rectangle with distinct positive integers such that elements in each column increase, reading from bottom to top, then we let $\operatorname{red}(F)$ denote the element of $\mathcal{F}_{n, k}$ which results from $F$ by replacing the $i$ th smallest element of $F$ by $i$. For example, Figure 2 pictures a filling $F$ with its corresponding reduced filling, $\operatorname{red}(F)$.

\begin{tabular}{|c|c|c|}
\hline 9 & 17 & 20 \\
\hline 7 & 13 & 15 \\
\hline 2 & 8 & 10 \\
\hline
\end{tabular}$\stackrel{\text { red }}{\longrightarrow} \quad$\begin{tabular}{|c|c|c|}
\hline 4 & 8 & 9 \\
\hline 2 & 6 & 7 \\
\hline 1 & 3 & 5 \\
\hline
\end{tabular}

Fig. 2: An example of $\operatorname{red}(F)$.

If $F \in \mathcal{F}_{n, k}$ and $1 \leq c_{1}<\cdots<c_{j} \leq n$, then we let $F\left[c_{1}, \ldots, c_{j}\right]$ be the filling of the $k \times j$ rectangle where the elements in column $a$ of $F\left[c_{1}, \ldots, c_{j}\right]$ equal the elements in column $c_{a}$ in $F$ for $a=1, \ldots, j$. We can then extend the usual pattern matching definitions from permutations to elements of $\mathcal{F}_{n, k}, \mathcal{C}_{=k[n-1]}$, or $\mathcal{S T}\left(n^{k}\right)$ as follows.

Definition 2. Let $P$ be an element of $\mathcal{F}_{j, k}\left(\mathcal{C}_{=k[j-1]}, \mathcal{S} \mathcal{T}\left(j^{k}\right)\right)$ and $F \in \mathcal{F}_{n, k}\left(\mathcal{C}_{=k[n-1]}, \mathcal{S} \mathcal{T}\left(n^{k}\right)\right)$ where $j \leq n$. Then we say

1. $P$ occurs in $F$ if there are $1 \leq i_{1}<i_{2}<\cdots<i_{j} \leq n \operatorname{such}$ that $\operatorname{red}\left(F\left[i_{1}, \ldots, i_{j}\right]\right)=P$,

2. $F$ avoids $P$ if there is no occurrence of $P$ in $F$, and

3. there is a $P$-match in $F$ starting at position $i$ if $\operatorname{red}(F[i, i+1, \ldots, i+j-1])=P$.

We let $P$-mch $(F)$ denote the number of $P$-matches in $F$. For example, if we consider the fillings $P \in \mathcal{F}_{3,3}$ and $F, G \in \mathcal{F}_{6,3}$ shown in Figure 3 , then it is easy to see that there are no $P$-matches in $F$ but there is an occurrence of $P$ in $F$ since $\operatorname{red}(F[1,2,5])=P$. Also, there are $2 P$-matches in $G$ starting at positions 1 and 2, respectively, so $P$ - $\operatorname{mch}(G)=2$.

We say that an element $P$ in $\mathcal{F}_{j, k}\left(\mathcal{C}_{=k[n-1]}, \mathcal{S T}\left(n^{k}\right)\right)$ has the minimal overlapping property or is minimally overlapping if the smallest $i$ such that there exists an $F \in \mathcal{F}_{i, k}\left(\mathcal{C}_{=k[i-1]}, \mathcal{S T}\left(i^{k}\right)\right)$ with $P-\operatorname{mch}(F)=2$ is $2 j-1$. Thus if $P$ has the minimal overlapping property, then two consecutive $P$ matches can only overlap in a single column, namely the last column of the first match and first column of the second match. We say that $P$ is overlapping if $P$ is not minimal overlapping.

If $P \in \mathcal{F}_{j, k}\left(\mathcal{C}_{=k[j-1]}, \mathcal{S T}\left(j^{k}\right)\right)$ has the minimal overlapping property, then the smallest $i$ such that there exists an $F \in \mathcal{F}_{i, k}\left(\mathcal{C}_{=k[i-1]}, \mathcal{S T}\left(i^{k}\right)\right)$ such that $P-\operatorname{mch}(F)=n$ is $n(j-1)+1$. If $P \in \mathcal{F}_{j, k}$, we let $\mathcal{M P}_{P, n(j-1)+1}$ denote the set of all $F \in \mathcal{F}_{n(j-1)+1, k}$ such that $P$-mch $(F)=n$ 


$$
\begin{aligned}
& P \quad \begin{array}{|l|l|l|}
\hline 3 & 6 & 9 \\
\hline 2 & 5 & 8 \\
\hline 1 & 4 & 7 \\
\hline
\end{array}
\end{aligned}
$$

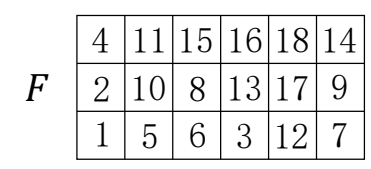

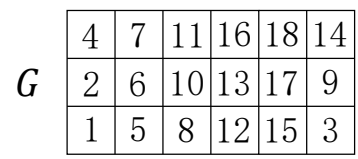

Fig. 3: Examples of $P$-matches and occurrences of $P$.

and set $\mathrm{mp}_{P, n(j-1)+1}=\left|\mathcal{M} \mathcal{P}_{P, n(j-1)+1}\right|$. If $P \in \mathcal{C}_{=k[j-1]}$, we let $\mathcal{C} \mathcal{M P} \mathcal{P}_{P, n(j-1)+1}$ denote the set of all $F \in \mathcal{C}_{=k[n(j-1)+1]}$ such that $P-\operatorname{mch}(F)=n$ and set $c \mathrm{mp}_{P, n(j-1)+1}=\left|\mathcal{C M P}_{P, n(j-1)+1}\right|$. If $P \in \mathcal{S T}\left(j^{k}\right)$, we let $\mathcal{S T} \mathcal{M} \mathcal{P}_{P, n(j-1)+1}$ denote the set of all $F \in \mathcal{S T}\left((n(j-1)+1)^{k}\right)$ such that $P-\operatorname{mch}(F)=n$ and set $s \operatorname{mp}_{P, n(j-1)+1}=\left|\mathcal{S T} \mathcal{T} \mathcal{P P}_{P, n(j-1)+1}\right|$. In each case we shall call an $F \in \mathcal{M P}_{P, n(j-1)+1}\left(F \in \mathcal{C} \mathcal{M} \mathcal{P}_{P, n(j-1)+1}, F \in \mathcal{S} \mathcal{T} \mathcal{M} \mathcal{P}_{P, n(j-1)+1}\right)$ a maximum packing for $P$.

Given $P \in \mathcal{F}_{j, k}$, let

$$
A_{P}(x, t)=\sum_{n \geq 0} \frac{t^{n}}{(k n) !} \sum_{F \in \mathcal{F}_{n, k}} x^{P-\operatorname{mch}(F)}
$$

Clearly, when $x=0$,

$$
A_{P}(0, t)=\sum_{n \geq 0} \frac{t^{n}}{(k n) !}\left|\left\{F \in \mathcal{F}_{n, k}: P-\operatorname{mch}(F)=0\right\}\right| .
$$

For $P, Q \in \mathcal{F}_{j, k}$, if $A_{P}(0, t)=A_{Q}(0, t)$, we say $P$ and $Q$ are c-Wilf equivalent. If $A_{P}(x, t)=A_{Q}(x, t)$, we say $P$ and $Q$ are strongly c-Wilf equivalent. In [10], in the case where $k=1$, Nakamura conjectured that if two permutations are c-Wilf equivalent then they are also strongly c-Wilf equivalent. Harmse and Remmel gave a similar conjecture in [7] when $k \geq 2$. That is, they made the following conjecture.

Conjecture 3. $P, Q \in \mathcal{F}_{n, k}$ are $c$-Wilf equivalent if and only if $P$ and $Q$ are strongly $c$-Wilf equivalent.

It has been shown that the conjecture holds for minimal overlapping patterns and the first and the last column of a pattern determines which c-Wilf equivalence class it belongs to (see [2], [3], [4], and [7]). The key to proving such results is to prove an analogue to Theorem 1 . This was done by Harmse and Remmel [7] who proved the following theorem for minimal overlapping patterns in $\mathcal{F}_{j, k}$.

Theorem 4. Suppose that $k \geq 2, j \geq 2$, and $P \in \mathcal{F}_{j, k}$ has the minimal overlapping property. Then

$$
A_{P}(t, x)=\frac{1}{1-\left(\frac{t}{k !}+\sum_{n \geq 1} \frac{t^{n(j-1)+1}}{(k(n(j-1)+1) !}(x-1)^{n} m p_{P, n(j-1)+1}\right)} .
$$

One can prove analogous results for minimal overlapping patterns $P$ in $\mathcal{C}_{=k[j-1]}$ and for minimal overlapping patterns $P$ in $\mathcal{S T}\left(j^{k}\right)$. However, such results can no longer be expressed just in terms of maximum packings, but require a more sophisticated concept of generalized maximum packing. This will be the subject of a forthcoming paper [11].

The main focus of this paper is the following questions. 
How many minimal overlapping patterns are there in $\mathcal{F}_{n, k}$ ?

How many minimal overlapping patterns are there in $\mathcal{S} \mathcal{T}\left(n^{k}\right)$ ?

How many minimal overlapping patterns are there in $\mathcal{C}_{=k[n-1]}$ ?

We let $M_{n, k}\left(S T M_{n, k}, C M_{n, k}\right)$ denote the number of minimal overlapping patterns in $\mathcal{F}_{n, k}\left(\mathcal{S T}\left(n^{k}\right)\right.$, $\mathcal{C}_{=k[n-1]}$. Then we will be interested in the following quantities:

$$
\begin{aligned}
a_{n, k} & =\frac{M_{n, k}}{\left|\mathcal{F}_{n, k}\right|}, \\
b_{n, k} & =\frac{S T M_{n, k}}{\left|\mathcal{S T}\left(n^{k}\right)\right|}, \text { and } \\
c_{n, k} & =\frac{C M_{n, k}}{\left|\mathcal{C}_{=k[n-1]}\right|} .
\end{aligned}
$$

Bóna studied the behavior of $a_{n, 1}$ in [1]. In [1], Bóna proved that $a_{n, 1} \geq 3-e \approx 0.2817$. That is, he proved that at least $28.17 \%$ of the permutations in $S_{n}$ are minimimal overlapping. Moreover, he proved that $\lim _{n \rightarrow \infty} a_{n, 1}$ exists. The main goal of this paper is to prove similar results for $a_{n, k}, b_{n, k}$, and $c_{n, k}$.

The outline of this paper is as follows. In Section 2, we shall give general lower bound for $a_{n, k}$ for all $k$. In Section 3, we shall give formulas for $M_{n, k}$ and $a_{n, k}$. In Section 4, we shall study the asymptotic behavior of $\left\{a_{n, k}\right\}$ for fixed $k$. In Section 5, we shall analyze $b_{n, k}$ and in Section 6, we shall analyze $c_{n, k}$. In section 7, we shall briefly discuss some open questions about the problem of finding the number of minimal overlapping permutations that start with a given initial segment.

\section{Lower Bound for $a_{n, k}$}

Bóna proved that the probability that a randomly selected pattern in $\mathcal{F}_{n, 1}$ is minimal overlapping is at least $3-e \approx 0.282$, that is, $L_{1}=3-e$ is a lower bound for $\left\{a_{n, 1}\right\}_{n \geq 1}$, independent on $n$. Based on Bóna's idea in [1], we are able to find a basic lower bound $L_{k}$ for $\left\{a_{n, k}\right\}_{n \geq 1}$ for any given $k \geq 1$.

We say that $P \in \mathcal{F}_{n, k}$ is overlapping at position $i$ if $\operatorname{red}(P[1, \ldots, i])=\operatorname{red}(P[n-i+1, \ldots, n])$. That is, the reduction of the first $i$ columns of $P$ is equal to the reduction of the last $i$ columns of $P$. Suppose $P \in \mathcal{F}_{n, k}$ is overlapping, then there exists integer $i, 2 \leq i \leq n-1$ such that $P$ is overlapping at position $i$. Furthermore, one can easily check that $P$ is overlapping if and only if there exists integer $i, 2 \leq i<\frac{n}{2}+1$ such that $P$ is overlapping at position $i$. Let $E_{i}$ be the event that $P$ is overlapping at position $i$. If $2 \leq i \leq \frac{n}{2}+1$, the probability that $E_{i}$ happens $\operatorname{Pr}\left(E_{i}\right)=\frac{(k !)^{i}}{(i k) !}$. That is, we can partition the elements of $\mathcal{F}_{n, k}$ by the set of elements that lie in the first $i$ columns of $P$ and the set of elements that lie in the last $i$ columns of $P$. Once the filling of the first $i$ columns of $P$ is fixed, there are $\frac{(i k) !}{(k !){ }^{i}}$ ways to arrange the elements in the last $i$ columns and only one of them is favorable. If $\frac{n}{2}<i<\frac{n}{2}+1$, $\operatorname{Pr}\left(E_{i}\right) \leq \frac{(k !)^{i}}{(i k) !}$. Then the probability that a randomly selected pattern in $\mathcal{F}_{n, k}$ is overlapping is bounded by

$$
\operatorname{Pr}\left(\bigcup_{2 \leq i<\frac{n}{2}+1} E_{i}\right) \leq \sum_{2 \leq i<\frac{n}{2}+1} \operatorname{Pr}\left(E_{i}\right) \leq \sum_{2 \leq i<\frac{n}{2}+1} \frac{(k !)^{i}}{(i k) !}<\sum_{i \geq 2} \frac{(k !)^{i}}{(i k) !} .
$$

Then for a fixed $k$, we get a lower bound $L_{k}$ for $\left\{a_{n, k}\right\}_{n \geq 1}$. 
Theorem 5.

$$
L_{k}=1-\sum_{i \geq 2} \frac{(k !)^{i}}{(i k) !} .
$$

Then by Theorem 5, we can compute $L_{1}$ and $L_{2}$ directly.

$$
\begin{aligned}
L_{1} & =1-\sum_{i \geq 2} \frac{1}{i !}=1-(-2+e)=3-e \approx 0.282 . \\
L_{2} & =1-\sum_{i \geq 2} \frac{(2 !)^{i}}{(2 i) !}=3-\sum_{i \geq 0} \frac{2^{i}}{(2 i) !}=3-\frac{1}{2}\left(\sum_{i=0} \frac{(\sqrt{2})^{i}}{i !}+\sum_{i=0} \frac{(-\sqrt{2})^{i}}{i !}\right) \\
& =3-\frac{1}{2}\left(e^{\sqrt{2}}+e^{-\sqrt{2}}\right)=3-\cosh (\sqrt{2}) \approx 0.822 .
\end{aligned}
$$

The results above indicate there are at least $28.2 \%$ of patterns in $\mathcal{F}_{n, 1}$ that are minimal overlapping, and at least $82.2 \%$ of patterns in $\mathcal{F}_{n, 2}$ that are minimal overlapping, no matter what $n$ is.

Moreover, by observing Equation (3), one can easily find that the sequence $\left\{L_{k}\right\}$ is monotone increasing. It agrees with our intuition because a pattern is minimal overlapping as long as there exists one row whose reduction is minimal overlapping and, hence, more rows means higher chance to be minimal overlapping. Also, since $\left\{L_{k}\right\}$ is bounded by 1 , the sequence converges.

\section{Theorem 6.}

$$
\lim _{k \rightarrow \infty} L_{k}=1
$$

Proof: Since

$$
L_{k}=1-\sum_{i \geq 2} \frac{(k !)^{i}}{(i k) !}
$$

to show (4) it suffices to show

$$
\lim _{k \rightarrow \infty} \sum_{i \geq 2} \frac{(k !)^{i}}{(i k) !}=0
$$

For $i \geq 2$,

$$
\lim _{k \rightarrow \infty} \frac{(k !)^{i}}{(i k) !}=0, \quad\left|\frac{(k !)^{i}}{(i k) !}\right| \leq \frac{1}{i^{2}} \quad \text { and } \quad \sum_{i \geq 2} \frac{1}{i^{2}}<\infty .
$$

Then by the dominated convergence theorem,

$$
\lim _{k \rightarrow \infty} \sum_{i \geq 2} \frac{(k !)^{i}}{(i k) !}=\sum_{i \geq 2} \lim _{k \rightarrow \infty} \frac{(k !)^{i}}{(i k) !}=0 .
$$

$\square$ Thus we have the following corollary.

Corollary 7. For a given n,

$$
\lim _{k \rightarrow \infty} a_{n, k}=1
$$

Now we see no matter how large $n$ is, almost every pattern in $\mathcal{F}_{n, k}$ is minimal overlapping as long as $k$ is large enough. Another fact worth mentioning is that $\left\{L_{k}\right\}$ converges to 1 rapidly. By $33, L_{1} \approx 0.282$, $L_{2} \approx 0.822, L_{3} \approx 0.950, L_{4} \approx 0.986, L_{5} \approx 0.996, \cdots$. 


\section{Formulas for $M_{n, k}$ and $a_{n, k}$}

It follows from our observations in the previous section that a pattern $P \in \mathcal{F}_{n, k}$ is overlapping if and only if there exists a unique integer $i$ such that $P$ is overlapping at position $i$ and the reduction of the first $i$ columns of $P$ is minimally overlapping with $2 \leq i<\frac{n}{2}+1$. The basic idea of obtaining the formula for $M_{n, k}$, the number of minimal overlapping patterns in $\mathcal{F}_{n, k}$, is to subtract the number of overlapping patterns from the total number.

Assuming $n$ is fixed, we separate the discussion of $M_{n, k}$ into two cases.

Case $1 . k$ is an even number.

$$
\begin{aligned}
M_{n, k}= & \left(\begin{array}{c}
n k \\
k, k, \ldots, k
\end{array}\right)-M_{2, k}\left(\begin{array}{c}
n k \\
2 k, 2 k
\end{array}\right)\left(\begin{array}{c}
(n-4) k \\
k, k, \cdots, k
\end{array}\right)-M_{3, k}\left(\begin{array}{c}
n k \\
3 k, 3 k
\end{array}\right)\left(\begin{array}{c}
(n-6) k \\
k, k, \cdots, k
\end{array}\right) \\
& -M_{4, k}\left(\begin{array}{c}
n k \\
4 k, 4 k
\end{array}\right)\left(\begin{array}{c}
(n-8) k \\
k, k, \ldots, k
\end{array}\right)-\cdots-M_{\frac{n}{2}, k}\left(\begin{array}{c}
n k \\
\frac{1}{2} n k, \frac{1}{2} n k
\end{array}\right) \\
= & \frac{(n k) !}{(k !)^{n}}-\sum_{i=2}^{\frac{n}{2}} M_{i, k}\left(\begin{array}{c}
n k \\
i k, i k
\end{array}\right) \frac{((n-2 i) k) !}{(k !)^{n-2 i}} .
\end{aligned}
$$

The only significant difference between the even case and the odd case is the term corresponding to patterns overlapping at position $\frac{n+1}{2}$.

Case 2. $n$ is an odd number.

$$
\begin{aligned}
M_{n, k}= & \left(\begin{array}{c}
n k \\
k, k, \ldots, k
\end{array}\right)-M_{2, k}\left(\begin{array}{c}
n k \\
2 k, 2 k
\end{array}\right)\left(\begin{array}{c}
(n-4) k \\
k, k, \cdots, k
\end{array}\right)-M_{3, k}\left(\begin{array}{c}
n k \\
3 k, 3 k
\end{array}\right)\left(\begin{array}{c}
(n-6) k \\
k, k, \cdots, k
\end{array}\right) \\
& -\cdots-M_{\frac{n-1}{2}, k}\left(\begin{array}{c}
n k \\
\frac{1}{2}(n-1) k, \frac{1}{2}(n-1) k
\end{array}\right)-\sum_{P \in \mathcal{M}_{\frac{n+1}{2}, k}} \operatorname{mp}_{P, n} \\
= & \frac{(n k) !}{(k !)^{n}}-\sum_{i=2}^{\frac{n-1}{2}} M_{i, k}\left(\begin{array}{c}
n k \\
i k, i k
\end{array}\right) \frac{((n-2 i) k) !}{(k !)^{n-2 i}}-\sum_{P \in \mathcal{M}_{\frac{n+1}{2}, k}} \mathrm{mp}_{P, n} .
\end{aligned}
$$

Dividing $M_{n, k}$ by $\left|\mathcal{F}_{n, k}\right|$, we get formula of $a_{n, k}$ as follows

\section{Theorem 8.}

$$
\begin{aligned}
& \text { If } n \text { is even, } \quad a_{n, k}=1-\sum_{i=2}^{\frac{n}{2}} a_{i, k} \frac{(k !)^{i}}{(i k) !} . \\
& \text { If } n \text { is odd, } \quad a_{n, k}=1-\sum_{i=2}^{\frac{n-1}{2}} a_{i, k} \frac{(k !)^{i}}{(i k) !}-b_{n, k},
\end{aligned}
$$

where $b_{n, k}=\frac{\sum_{P \in \mathcal{M}^{n+1}, k} m p_{P, n}}{\left|\mathcal{F}_{n, k}\right|}$.

It may seem that (6) and (7) gives us a way to recursively compute $a_{n, k}$, but unfortunately this is not the case. That is, while the even terms depend only on the previous values, the odd terms rely on both previous terms and $\sum_{P \in \mathcal{M}_{(n+1) / 2, k}} \mathrm{mp}_{P, n}$ which is difficult to compute. Indeed, we have no general way to compute $\mathrm{mp}_{P, n}$ for a fixed minimal overlapping $P$. 


\section{The limit of $a_{n, k}$}

In this section, we study the asymptotic behavior of $\left\{a_{n, k}\right\}_{n \geq 1}$, for any given integer $k \geq 1$. Throughout this section, we shall assume that $k$ is a fixed number greater than or equal to 1 . We let $\left\{d_{n}\right\}$ be the subsequence of $\left\{a_{n, k}\right\}_{n \geq 1}$ consisting of odd terms, ie., $d_{j}=a_{2 j-1, k}, j=1,2,3, \cdots$. We let $\left\{e_{n}\right\}$ be the subsequence consisting of even terms.

From equation 6 in Theorem 8 , we see $\left\{e_{n}\right\}$ is a monotone decreasing sequence and $a_{n, k}$ has lower bound $L_{k}$, hence $\left\{e_{n}\right\}$ is convergent. To show $a_{n, k}$ has a limit, we need to show $\left\{d_{n}\right\}$ converges to the limit of $\left\{e_{n}\right\}$. This will result in the following theorem.

Theorem 9. For a fixed integer $k \geq 1, \lim _{n \rightarrow \infty} a_{n, k}$ exists.

Proof: We only need to show the limit of $d_{n}$ equals the limit of $e_{n}$. Since $d_{n+1}=a_{2 n+1, k}$ and $e_{n}=a_{2 n, k}$, by (6) and (7), we have $e_{n}-d_{n+1}=b_{2 n+1, k}$.

$$
b_{2 n+1, k}=\frac{\sum_{P \in \mathcal{M}_{n+1, k}} \mathrm{mp}_{P, 2 n+1}}{\left|\mathcal{F}_{2 n+1, k}\right|} \leq \frac{M_{n+1, k}\left(\begin{array}{c}
(2 n+1) k \\
n k
\end{array}\right)}{\left|\mathcal{F}_{2 n+1, k}\right|}
$$

The inequality holds because that the fact that the reduction of the first $n+1$ columns of a pattern in $\mathcal{F}_{2 n+1, k}$ is minimal overlapping does not guarantee the pattern is overlapping at position $n+1$. In other words, it is a necessary condition that the reduction of the first $n+1$ columns is minimal overlapping but that it is not sufficient. Take patterns in $\mathcal{F}_{5,1}$ as an example. Assume the reduction of the first three numbers is 132 which is minimal overlapping. If the initial three numbers are 342 , we can put 51 in the end to make 34251 overlapping at the middle position. However, a pattern with prefix 342 can not be overlapping at position 3 . We use RHS for the right-handed side of (8).

$$
\begin{aligned}
\text { RHS } & =\frac{\left(\begin{array}{c}
(2 n+1) k \\
n k
\end{array}\right) M_{n+1, k}}{\left|\mathcal{F}_{2 n+1, k}\right|}=\frac{\left(\begin{array}{c}
(2 n+1) k \\
n k
\end{array}\right)\left|\mathcal{F}_{n+1, k}\right| a_{n+1, k}}{\left|\mathcal{F}_{2 n+1, k}\right|} \\
& =\frac{(2 n k+k) !(n k+k) !(k !)^{2 n+1}}{(n k) !(n k+k) !(k !)^{n+1}(2 n k+k) !} a_{n+1, k} \\
& =\frac{(k !)^{n}}{(n k) !} a_{n+1, k} \\
& =\frac{a_{n+1, k}}{\left|\mathcal{F}_{n, k}\right|}
\end{aligned}
$$

As $n$ goes to infinity, $\left|\mathcal{F}_{n, k}\right|$ goes to infinity and because $a_{n+1, k}$ is bounded by 1 ,

$$
\lim _{n \rightarrow \infty} e_{n}-d_{n+1}=\lim _{n \rightarrow \infty} b_{2 n+1, k}=0 .
$$

Therefore, $\lim _{n \mid \infty} d_{n}=\lim _{n \mid \infty} e_{n}$ and then hence $\lim _{n \rightarrow \infty} a_{n, k}$ exists. $\square$ Note that $\left|\mathcal{F}_{n, k}\right|$ increases rapidly as $n$ increases so that $a_{n, k}$ converges rapidly. The larger $k$ becomes, the faster $a_{n, k}$ converges.

Let $l_{k}$ be the limit of $a_{n, k}$ as $n$ goes to infinity. Using Monte Carlo methods, we computed approximate values for $l_{k}$ for $k=1,2,3,4: l_{1} \approx 0.364, l_{2} \approx 0.823, l_{3} \approx 0.949$ and $l_{4} \approx 0.986$. Comparing $L_{k}$ and $l_{k}$, we find they are very close. This is not a coincidence because (3) is almost the same as (6) and (7) and we know $a_{n, k}$ is close to 1 when $n$ is large. 


\section{Standard tableaux of rectangular shapes}

The set of all the standard Young tableaux of rectangular shape $n^{k}$ is denoted by $\mathcal{S} \mathcal{T}\left(n^{k}\right)$ and clearly it is a subset of $\mathcal{F}_{n, k}$. It is well-known that the cardinality of $\mathcal{S T}\left(n^{2}\right)$ is the $n$th Catalan number $\operatorname{Cat}(n)=$ $\frac{1}{n+1}\left(\begin{array}{c}2 n \\ n\end{array}\right)$. Then using the similar argument of Theorem 5. we have a lower bound for $b_{n, 2}$, where $b_{n, 2}$ is proportion of minimal overlapping patterns in $\mathcal{S} \mathcal{T}\left(n^{2}\right)$

$$
L_{2}^{S}=1-\sum_{i \geq 2} \frac{1}{\operatorname{Cat}(i)}
$$

To find the exact value of the lower bound, we must compute the sum $\sum_{i \geq 2} \frac{1}{\operatorname{Cat}(i)}$. Define a power series $f(x)$ as follows

$$
f(x)=\sum_{i \geq 1} \frac{1}{\operatorname{Cat}(i)} x^{i}
$$

Then

$$
x f^{\prime}(x)=x \sum_{i \geq 1} \frac{i}{\operatorname{Cat}(i)} x^{i-1}=\sum_{i \geq 1} \frac{i}{\operatorname{Cat}(i)} x^{i}
$$

By the recursion of Catalan numbers Cat $(i+1)=\frac{4 i+2}{i+2} \mathrm{Cat}(i)$,

$$
\begin{aligned}
x f^{\prime}(x) & =\sum_{i \geq 1} \frac{i+2-2}{\operatorname{Cat}(i)} x^{i}=\sum_{i \geq 1} \frac{i+2}{\operatorname{Cat}(i)} x^{i}-2 \sum_{i \geq 1} \frac{1}{\operatorname{Cat}(i)} x^{i}=\sum_{i \geq 1} \frac{4 i+2}{\operatorname{Cat}(i+1)} x^{i}-2 f(x) \\
& =\sum_{i \geq 1} \frac{4(i+1)-2}{\operatorname{Cat}(i+1)} x^{i}-2 f(x)=4 \sum_{i \geq 1} \frac{i+1}{\operatorname{Cat}(i+1)} x^{i}-2 \sum_{i \geq 1} \frac{1}{\operatorname{Cat}(i+1)} x^{i}-2 f(x) \\
& =\frac{4}{x}\left(x f^{\prime}(x)-x\right)-\frac{2}{x}(f(x)-x)-2 f(x)=4 f^{\prime}(x)-\frac{2 x+2}{x} f(x)-2 .
\end{aligned}
$$

So we obtain a first-order ordinary differential equation

$$
f^{\prime}(x)+\left(\frac{2 x+2}{x^{2}-4 x}\right) f(x)=\frac{2}{4-x} .
$$

Solving this differential equation 9 with the initial value condition $f(0)=0$ for $f(x)$ yields

$$
\frac{(x-10)(x-4) x+24 \sqrt{4 x-x^{2}} \arcsin \left(\frac{\sqrt{x}}{2}\right)}{(4-x)^{3}}
$$

Setting $x=1$ in this formula for $f(x)$ gives our next theorem.

\section{Theorem 10.}

$$
\sum_{i \geq 1} \frac{1}{\operatorname{Cat}(i)}=1+\frac{4 \sqrt{3} \pi}{27}
$$


Then by the above equation, we have the lower bound $L_{2}^{S}=1-\frac{4 \sqrt{3} \pi}{27} \approx 0.194$.

Similarly, for any given $k$, lower bounds for $b_{n, k}$, that is, lower bound for the proportion of minimal overlapping patterns in $\mathcal{S} \mathcal{T}\left(n^{k}\right)$ is

$$
L_{k}^{S}=1-\sum_{i \geq 2} \frac{1}{Y_{i^{k}}},
$$

where $Y_{i^{k}}=\left|\mathcal{S T}\left(i^{k}\right)\right|$ which could be counted by the hook-length formula. We computed the following approximations for $L_{k}$ for $k=3,4,5,6$.

$$
L_{3}^{S} \approx 0.774, \quad L_{4}^{S} \approx 0.926, \quad L_{5}^{S} \approx 0.976, \quad L_{6}^{S} \approx 0.992 .
$$

It is easy for one to show that $\lim _{k \rightarrow \infty} L_{k}^{S}=1$ which implies almost every pattern in $\mathcal{S} \mathcal{T}\left(n^{k}\right)$ is minimal overlapping as $k$ is very large.

Next we shall find an upper bound for the proportion of minimal overlapping standard tableaux of shape $n^{2}$. This is equivalent to finding a lower bound for the proportion of overlapping patterns. For a pattern $P \in \mathcal{S} \mathcal{T}\left(n^{2}\right), n \geq 3$, we only consider patterns where the reduction of the first two columns is the same as the reduction of the last two columns. There are only two cases for such overlapping patterns. For Case 1 (Figure 4), the number of such overlapping patterns in $\mathcal{S T}\left((n+2)^{2}\right)$ is equal to $\left|\mathcal{S T}\left(n^{2}\right)\right|$.

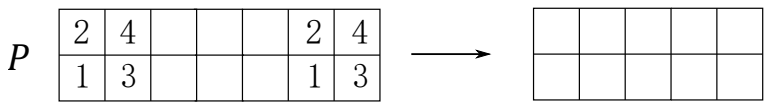

Fig. 4: Reducing via the patterns in the first two columns for Case 1

For Case 2 (Figure 5, the number of such overlapping patterns in $\mathcal{S T}\left((n+2)^{2}\right)$ is equal to the number of standard Young tableaux of skew shape $(n+2, n) /(2)$. It is easy to see that the number of standard

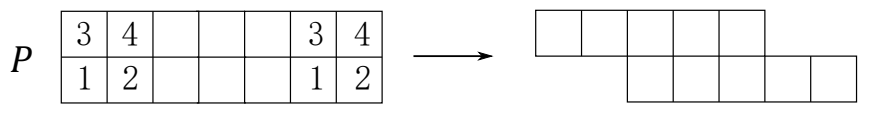

Fig. 5: Reducing via the patterns in the first two columns for Case 2

tableaux of shape $(n+2, n) /(2)$ is equal to the number of standard tableaux of shape $(n+2, n)$ where 1 and 2 lie in the first row. But then the number of standard tableaux of $(n+2, n)$ where 1 and 2 lie in the first row is equal to the number of standard tableaux of shape $(n+2, n)$ minus the number of standard tableaux of shape $(n+2, n)$ where 1 and 2 lie first column. But then the number of standard tableaux of shape $(n+2, n)$ where 1 and 2 lie first column is equal to the number of standard tableaux of shape $(n+1, n-1)$. Using the hook length formula for the number of standard tableaux, it follows that

$$
\begin{aligned}
|\mathcal{S T}(n+2, n) /(2)| & =|\mathcal{S T}(n+2, n)|-|\mathcal{S T}(n+1, n-1)| \\
& =\frac{3(2 n+2) !}{(n+3) ! n !}-\frac{3(2 n) !}{(n+2) !(n-1) !} \\
& =\frac{9 n^{2}+9 n+6}{(n+3)(n+2)(n+1)}\left(\begin{array}{c}
2 n \\
n
\end{array}\right) .
\end{aligned}
$$


Since the lower bound for number of overlapping patterns in $\mathcal{S T}\left((n+2)^{2}\right)$ is $\left|\mathcal{S T}\left(n^{2}\right)\right|+\mid \mathcal{S T}((n+$ $2, n) /(2)) \mid$, an upper bound for $b_{n+2,2}, n \geq 3$ is

$$
\begin{aligned}
U_{n+2,2}^{S} & =1-\frac{\left|\mathcal{S T}\left(n^{2}\right)\right|+|\mathcal{S T}((n+2, n) /(2))|}{\left|\mathcal{S T}\left((n+2)^{2}\right)\right|} \\
& =\frac{3 n^{2}+9 n}{8 n^{2}+16 n+6} .
\end{aligned}
$$

As $n$ goes to infinity, $U_{n+2,2}^{S}$ converges to 0.375 . Therefore, $\left\{b_{n, 2}\right\}$ is asymptotically between 0.194 and 0.375 .

\section{Generalized Euler permutations}

Elements in $\mathcal{C}_{=k[n-1]}$ are called generalized Euler permutations or $k$-up-down permutations. Note that 2-up-down permutations are usually called up-down permutations. For example, $\sigma=23581467$ is a 4-up-down permutation of length 8 and $\mu=152634$ is an up-down permutation permutation of length 6.

Next we give a lower bound $L_{k}^{E}$ for $\left\{c_{n, k}\right\}_{n \geq 2}$, that is, the proportion of minimal overlapping patterns in $\mathcal{C}_{=k[n-1]}$. Using essentially the same argument that we used to prove Theorem 5, we have a lower bound $L_{k}^{E}$

$$
L_{k}^{E}=1-\sum_{j \geq 2} \frac{1}{\left|\mathcal{C}_{=k[j-1]}\right|},
$$

where $\left|\mathcal{C}_{=k[j-1]}\right|$ is the number of $k$-up-down permutations of length $k j$. The generating function of $\left|\mathcal{C}_{=k[j-1]}\right|$ is

$$
\sum_{n \geq 0}\left|\mathcal{C}_{=k[j-1]}\right| \frac{t^{k n}}{(k n) !}=\frac{1}{\sum_{n \geq 0} \frac{(-1)^{n} t^{k n}}{(k n) !}}
$$

See Stanley's book, Chapter 3, page 389 [14]. It is apparent that for fixed $k, L_{k}^{E}$ converges so we can calculate numerical approximation

$$
\begin{aligned}
& L_{2}^{E}=1-\sum_{j \geq 2} \frac{1}{\left|\mathcal{C}_{=2[j-1]}\right|}=1-\frac{1}{5}-\frac{1}{61}-\frac{1}{1382}-\frac{1}{50521}-\cdots \approx 0.783, \\
& L_{3}^{E}=1-\sum_{j \geq 2} \frac{1}{\left|\mathcal{C}_{=3[j-1]}\right|}=1-\frac{1}{19}-\frac{1}{1513}-\frac{1}{315523}-\cdots \approx 0.947 \\
& L_{4}^{E}=1-\sum_{j \geq 2} \frac{1}{\left|\mathcal{C}_{=4[j-1]}\right|}=1-\frac{1}{69}-\frac{1}{33661}-\frac{1}{60376809}-\cdots \approx 0.985, \\
& L_{5}^{E}=1-\sum_{j \geq 2} \frac{1}{\left|\mathcal{C}_{=5[j-1]}\right|}=1-\frac{1}{251}-\frac{1}{750751}-\frac{1}{11593285251}-\cdots \approx 0.996 .
\end{aligned}
$$

It is easy for one to show that $\lim _{k \rightarrow \infty} L_{k}^{E}=1$ which means almost all patterns in $\mathcal{C}_{=k[n-1]}$ are minimal overlapping when $k$ is large. 
Next we will find an upper bound for $c_{n, 2}$. It is well-known that numbers of up-down permutations are Euler numbers. Suppose $E(n)$ is the $n$th Euler number, then $\left|\mathcal{C}_{=2[n-1]}\right|=E(2 n)$. By [15], the ratio of two adjacent Euler numbers have asymptotic estimation as follows

$$
\frac{E(n+1)}{E(n)} \sim \frac{2(n+1)}{\pi} .
$$

To find an upper bound of minimal overlapping patterns, we only need to find a lower bound for overlapping up-down permutations. Similar to Section 5, we only consider patterns where the reduction of the first two columns is the same as the reduction of the last two columns. In other words, we only consider prefixes and suffixes of length 4 in an up-down permutation and they have the same reduction. In [12], the number of such overlapping up-down permutations of length $2 n$ is given as

$$
13 E(2 n)-32 n E(2 n-1)+10 n(2 n-1) E(2 n-2),
$$

where $n \geq 4$. Hence we can get an upper bound for the percentage of minimal overlapping patterns in $\mathcal{C}_{=2[n-1]}$.

$$
U_{n, 2}^{E}=1-\frac{13 E(2 n)-32 n E(2 n-1)+10 n(2 n-1) E(2 n-2)}{E(2 n)} .
$$

Applying (11), we get an asymptotic upper bound

$$
U_{2}^{E}=\lim _{n \rightarrow \infty} U_{n, 2}^{E}=8 \pi-\frac{5}{4} \pi^{2}-12 \approx 0.795 .
$$

Therefore, $\left\{c_{n, 2}\right\}$ is asymptotically between 0.783 and 0.795 .

\section{Open questions}

A natural extension of our results would be to find the proportion of minimal overlapping patterns in $\mathcal{F}_{n, k}$ $\left(\mathcal{C}_{=k[n-1]}, \mathcal{S T}\left(n^{k}\right)\right)$ whose first $j$ columns is equal to some fixed $P \in \mathcal{F}_{j, k}$ among all the elements of $\mathcal{F}_{n, k}$ $\left(\mathcal{C}_{=k[n-1]}, \mathcal{S T}\left(n^{k}\right)\right)$ whose first $j$ columns is equal to $P$. For example, in the simplest case, we would be interested in the question of how many permutations in $S_{n}$ starting with $m$ are minimal overlapping.

It is not hard to see that for a fixed $m$, as $n$ approaches to infinity, there are at least $(3-e)(n-1)$ ! permutations in $S_{n}$ starting with $m$ are minimal overlapping. Similar to Theorem 9 , we can show that the proportion of minimal overlapping patterns in $S_{n}$ starting with $m$ converges as $n$ goes to infinity.

We have used Monte Carlo methods to estimate the limit of the proportion of minimal overlapping permutations starting among all permutations that start with $m$ for $m=1,2,3,4,5,6,7$. Our computations yielded the following estimates: $0.392,0.384,0.375,0.368,0.365,0.361$ and 0.358 respectively. Noting that the sequence is monotone decreasing, we ask whether this is true in general. That is, if $1 \leq a<b \leq\left\lceil\frac{n}{2}\right\rceil$, is it the case that as $n$ approaches infinity, is the proportion of minimal overlapping permutations in $S_{n}$ that start with $a$ among all the permutations of $S_{n}$ that start with $a$ greater than the proportion of minimal overlapping permutations in $S_{n}$ that start with $b$ among all the permutations of $S_{n}$ that start with $b$ ? 


\section{References}

[1] M. Bóna, Non-overlapping permutation patterns, Pure Math. and Applications, 22 (2011), 99-105.

[2] V. Dotsenko and A. Khoroshkin, Shuffle algebras, homology, and consecutive pattern avoidance, Algebra Number Theory, 7 (2013), 673-700.

[3] A.S. Duane and J.B. Remmel, Minimal overlapping patterns in colored permutations, Electronic J. Combinatorics, 18(2) (2011), P25, 34 pgs.

[4] S. Elizalde, The most and the least avoided consecutive patterns, Proc. Lond. Math. Soc. 106 (2013), 957-979

[5] S. Elizalde, A survey of consecutive patterns in permutations, to appear in Recent Trends in Combinatorics, IMA, 2015.

[6] J. Harmse, Pattern matching in column-strict fillings of rectangular arrays. PhD thesis, UC San Diego, 2011.

[7] J. Harmse and J.B. Remmel, Patterns in column strict fillings of rectangular arrays, Pure Math. and Applications, 22 (2011), 131-171.

[8] A. Mendes and J.B. Remmel, Permutations and words counted by consecutive patterns, Advances Appl. Math., 37 4, (2006), 443-480.

[9] A. Mendes and J.B. Remmel, Counting with symmetric functions, Developments in Mathematics, Springer, 2015.

[10] B. Nakamura, Computational approaches to consecutive pattern avoidance in permutations, Pure Math. and Applications, 22 (2011), 253-268.

[11] R. Pan and J.B. Remmel, Generalized cluster method for consecutive pattern matching in restricted combinatorial objects, in preparation.

[12] R. Pan and J.B. Remmel, Counting alternating permutations with prefix and suffix, in preparation.

[13] J.B. Remmel, Consecutive up-down patterns in up-down permutations, Electronic J. Combinatorics, 21(3) (2014), \#P3.2.

[14] R.P. Stanley, Enumerative combinatorics, vol. 1, Cambridge Studies in Advanced Mathematics, Cambridge University Press, Cambridge, 1997.

[15] R. P. Stanley, A survey of alternating permutations, in Combinatorics and graphs, AMS, Providence, RI, 2010, 165-196. 